\title{
PARA UN CATALOGO DE «SIGNUM TABELLIONIS" DE LA CORONA DE ARAGON: ALGUNOS NOTARIOS DE LAS CIUDADES DE VALENCIA Y ALZIRA DEL SIGLO XIII
}

\author{
Virginia M. ${ }^{a}$ Cuñat Ciscar
}

En 1978 Luisa d'Arienzo ponía en evidencia (1) la escasa atención que habían suscitado los estudios sobre el "signum tabellionis" en todo el territorio de la Corona de Aragón. Desde entonces hasta ahora —ver la recopilación bibliográfica del profesor Trenchs (2) — sólo se ha añadido a la bibliografía señalada por ella, su artículo (3) y el trabajo de Ana M. ${ }^{a}$ Navarro Escolano.

Consideramos que las propuestas presentadas por la profesora Arienzo siguen siendo válidas: reunir en un sólo catálogo los signos notariales y, lo que es más importante, estudiar su evolución y la unción que han recibido a través de los siglos (concretando el estudio en la Corona de Aragón) como medio de convalidación del documento público o privado y más como manifestación visible de la fe pública que es depositada en el notario.

Por ello en el presente estudio damos a conocer una serie de signum tabellionis pertenecientes a notarios públicos de las ciudades de Valencia y Alzira, todos ellos del siglo XIII, de la época posterior a la conquista de Jaime 1 .

Ninguno de los notarios que estudiamos pertenecen a la Cancillería Real, aunque alguno esté relacionado con el monarca en asuntos de índole comercial (compra-venta de bienes realizada por el rey) lo que hemos constatado en la recopilación de regestas de cada notario. Todas estas regestas han sido incluidas en este trabajo con el fin de completar mediante noticias biográficas y del ejercicio de la profesión la reproducción de los signum tabellionis que presentamos. 
Para realizar las reproducciones hemos tomado como figura básica la que aparece en los pergaminos elaborados por dichos notarios y pertenecientes a los fondos del Archivo Histórico Nacional, sección Clero, y dentro de ella, los agrupados en Bernardos en las carpetas números $3.360,3.362,3.363$ y 3.365 .

Completamos el estudio con un gráfico temporal de ejercicio de su profesión.

\section{CUADRO CRONOLOGICO}

ASTRUC, Arnoldus

BERENGARIUS, Egidius

ERMENGAUD !, GULLLMUS

COLUMB I, GuilleLmus

IACCA, Arnoldus de

IACCA, Dominicus de

IACCA, Guillelmus de

LUzano, Arraldus de

PAULI, Petrus

RABACIE, I anvarius

SANTA CRUCE, PEtrus de

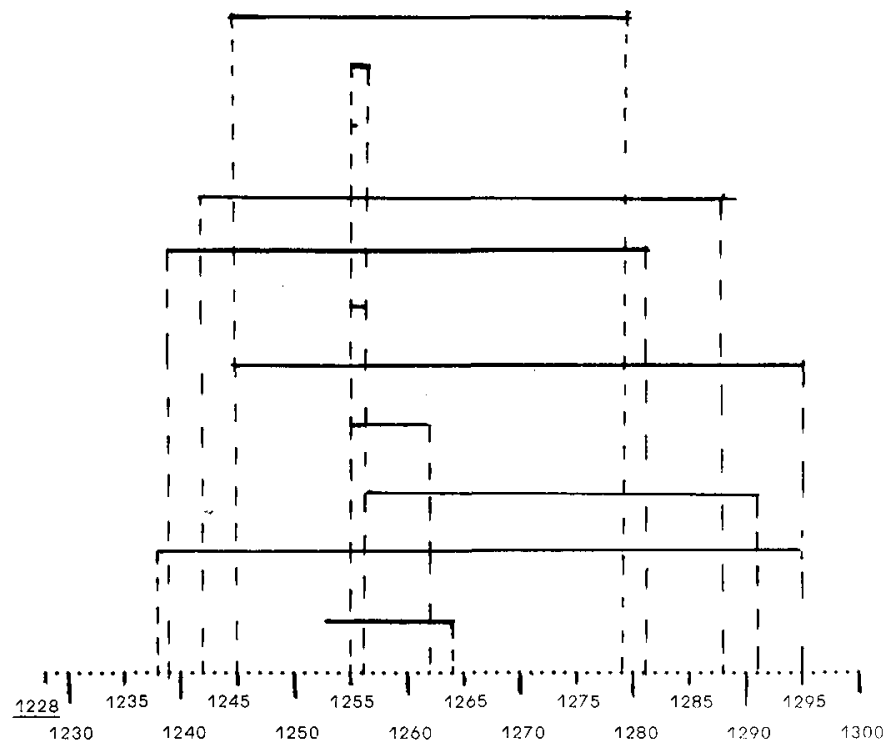




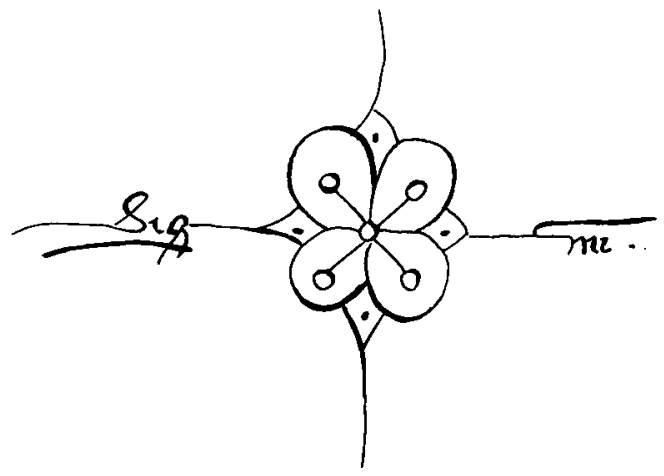

I. ASTRUC, Arnaldus (ASTRUC, Arnau) -Notario de Valencia-Regestas $n .^{\circ}$ $19,22,24,25,28,33,37$ y 41 .

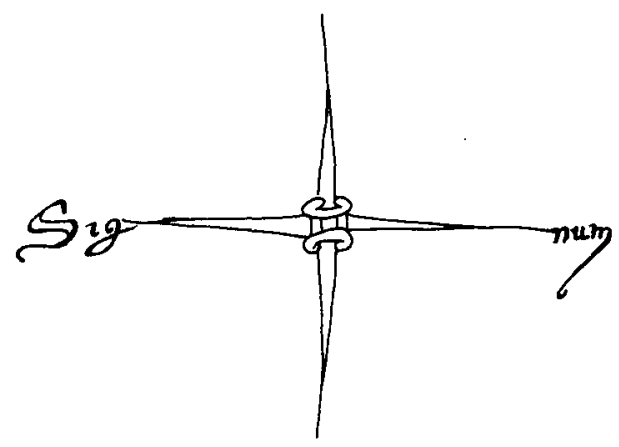

II. BERENGARIUS, Egidius (BERENGUER, Gil) - Notario de Valencia-Regestas $n .^{\circ} 12$.

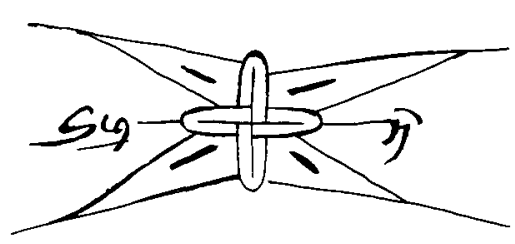

III. ERMENGAUDI, GuillelmUS (ARMENGOL, Guillem) - Notario de AlziraRegestas $n .^{\circ} 8,14,39$. 


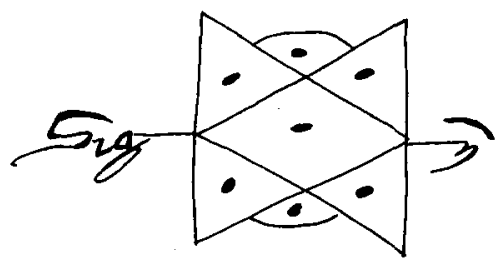

IV. COLUMBI, Guillelmus (COLOM, Guillem) - Notario de Alzira-Regestas $n .^{\circ} 3,5,6,7,36$ y 48 .

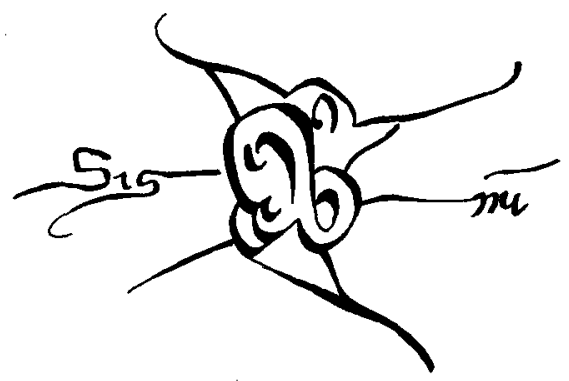

V. IACCA, Arnaldus de (JACA, Arnau de) -Notario de Valencia-Regestas n. 2,23 y 29.

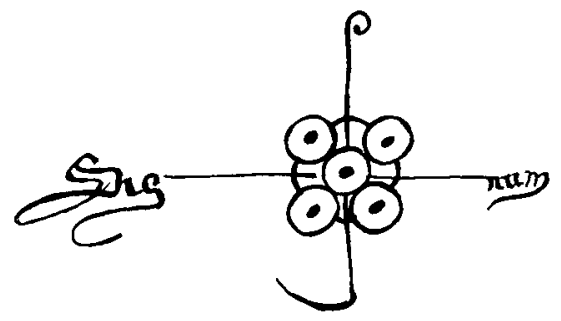

VI. IACCA, Dominicus de (JACA, Domenec de) -Notario de Valencia-Regestas $n .^{\circ} 9$ y 11 . 


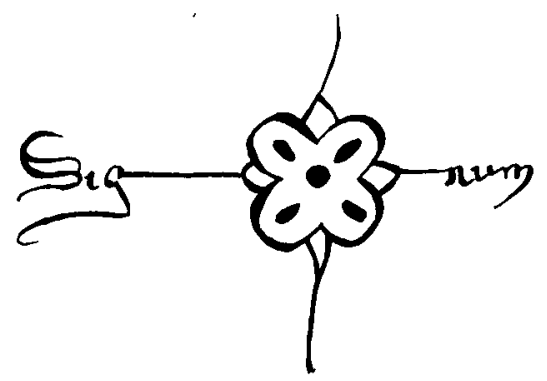

VII. IACCA, Guillemus de (JACA, Guillem de) -Notario de Valencia-Regestas n. ${ }^{\circ} 13,16,20$ y 55.

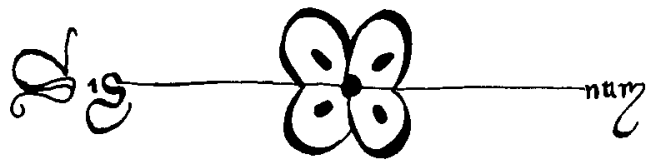

VIII. LUZANO, Arnaldus de (LLUSA, Arnau de) - Notario de Valencia-Regestas $n .^{\circ} 10,18,21$ y 26.

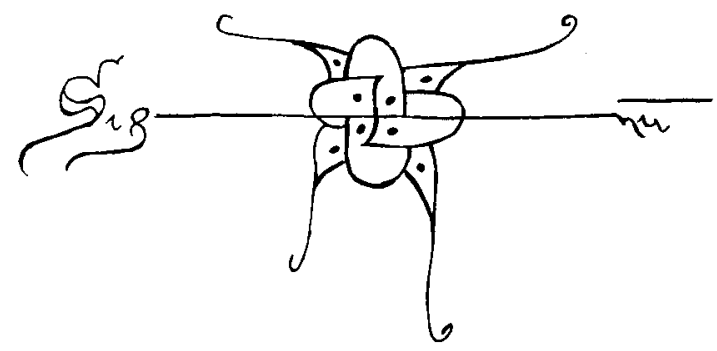

IX. PAULI, Petrus (PAU, Pere) - Notario de Valencia-Regestas $n .^{\circ} 15,17,31$, 34 y 54 . 


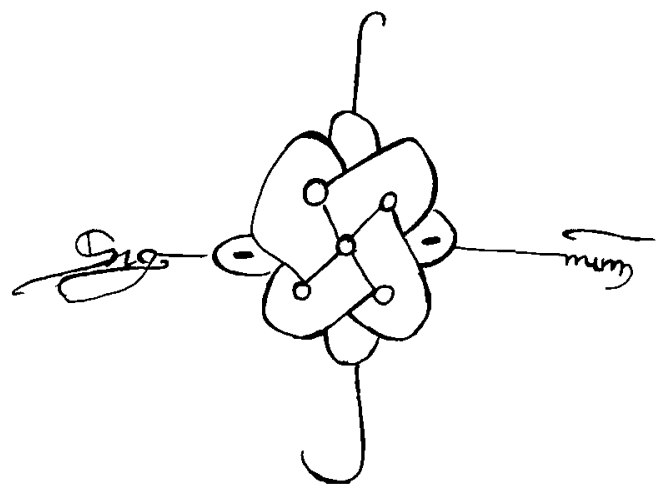

X. RABACIE, lanuarius (RABAGA, Janer) -Notario de Valencia-Regestas $n .^{\circ}$ $1,32,35,38,40,41,42,43,44,45,46,47,49,50,51,52,53,55$ y 57.

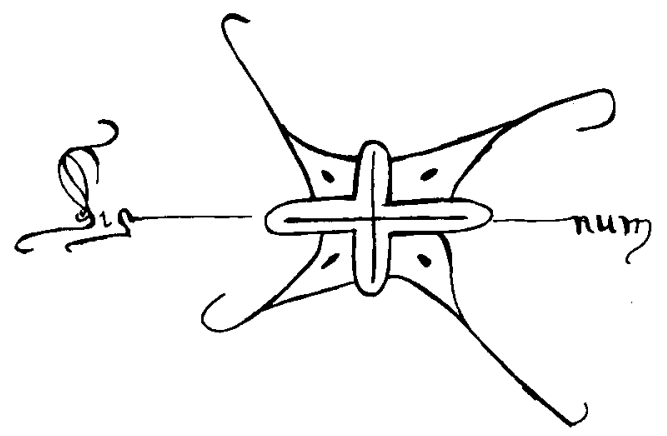

XI. SANTA CRUCE, Petrus de (SANTA CREU, Pere de) - Notario de AlziraRegestas $n .^{\circ} 7$ y 27. 
1

1238, octubre, 18. Valencia

Jaime I concede a Vidal, obispo de Huesca, la alquería de Alborayeg a cambio de la de Alcudia que le había otorgado con anterioridad. Janer Rabaça es uno de los testigos.

A.H.N. Clero. Valldigna. Bernardos. Carp. 3.359 n. ${ }^{\circ} 7$

REG. BARRAGAN GOMEZ, M. doc. 2

2

1239 , diciembre, 28

Jaime I concede a Berenguer de Novo unas casas en Valencia en la iglesia de Santa María. Notario Guillermo Scriba. Arnau de Jaca, notario de Valencia realiza en esa fecha el documento de traslado.

A.C.V. leg. 26: 7

REG. OLMOS CANALDA n. ${ }^{\circ} 41 \mathrm{a}$

3

1242, mayo, 29

Jaime I concede a Guillém Colom, Ramón de Graus, Guillém Gantaroya y a sus compañeros veinticinco jovadas de doce cahizadas de la medida de Valencia, además veinticinco casas.

A.C.A. R. f. 19

ED. LIBRE $n .^{\circ} 2.063$

4

1245, agosto, 17. Lérida

Jaime I concede ocho yugadas de tierra junto al rio Palmar y un solar en el arrabal de Denia, para edificar casas, a Guillermo de Baç y a su orden a condición de tener en la villa de Denia un Hospital.

A.H.N. Clero. Merced. Puig. Carp. 3.193 n. ${ }^{\circ} 4$

REG. VEDREÑO ALBA, M. ${ }^{a}$ C. doc. 18.

5

1248, junio, 26

Jaime I concede a Guillém Colom de unas casas en la algorfa de Játiva y una jovada en su término, además de otra jovada junto al puig para cultivar viñas.

A.C.A. R. f. 62

ED. LIBRE n. ${ }^{\circ} 2574$

6

1249, agosto, 8

Jaime I concede a Guillém Colom, Arnau Caçola y a Guillém Çasena de cuatro jovadas de viña a cada uno y además hanegadas de 
huerto en Andarella, Ondara, Benimantell, rafal de Guadalest, Benidechi y Benibatha, alquerias de Guadalest. $Y$ a cincuenta y seis compañeros suyos cuatro jovadas de tierra a cada uno en Maura, Miro, Benifato y Zaneta, alquerias de Valle de Guadalest, y si no hubiera suficiente en el mismo valle que estuvieran próximas.

A.C.A. R. f. 81

ED. LIBRE n. ${ }^{\circ} 2.934$.

7

1253, agosto, 20. Alzira

Documento de traslado de la donación que en Morella el 24 de marzo de 1250 Jaime I hizo a Guillém de Rocafull de unas casas y toda la heredad que su hermano, Bertrán de Rocafull, tenía en Alzira, Corbera y otros lugares del reino de Valencia. Pere Andreu es el primer notario. Guillém Colom es uno de los testigos. Pere de Santa Creu, notario de Alzira, realiza el documento de traslado.

A.H.N. Clero. Valldigna. Bernardos. Carp. 3.360 n. ${ }^{\circ} 6$

ED. BARRAGAN GOMEZ, M. doc. 13.

8

1255, enero, 14. Alzira

Jimeno de Alvero vende unas casas situadas en Alzira a Domenec de Cavall. Guillém Armengol, notario.

A.H.N. Clero. Validigna. Bernardos. Carp. 3.361 n. 1.

9

1255, octubre, 7. Valencia

Guillém de Aguilar, ciudadano de Valencia, vende una mazmudina y media en Rascaña (Valencia) a Arnaldo Cardona. Domenec de Jaca, notario.

A.R.V. caja 3, perg. 14.

10

1256

Testamento de Fernando Garcés de Roda, señor de Sot de Xera. Notario Arnau de Llusâ.

A.C.V. leg. 15: 1.

REG. OLMOS CANALDA n. 249.

11

1256, agosto, 13. Valencia

Guillermo de Loarre, portero del rey, vende una heredad que posee en Benivaire (Valencia) a Domenec de Cavall. Domenec de Jaca, notario.

A.H.N. Clero. Valldigna. Bernardos. Carp. 3.361 n. ${ }^{\circ} 2$. 
Pedro de Torres Blancas y su mujer venden a Arnau de Romaní unas tierras que poseen en Ollería (Valencia). Berenguer Gil, notario. A.H.N. Clero. Valldigna. Bernardos. Carp. 3.361 n. ${ }^{\circ} 4$.

13

1256, noviembre, 4. Valencia

Testamento del canónigo de la catedral Bertrán Teruel. Guillém de Jaca, notario.

A.C.V. perg. 5.012

REG. OLMOS CANALDA $n .^{\circ} 268$.

14

1256, diciembre, 6. Valencia

Guillelma, mujer de Pedro Carxo, vende unas tierras que posee en la alqueria de Benivaire (Alzira) a Juan López de Sartore. Berenguer Gil, notario.

A.H.N. Clero. Valldigna. Bernardos. Carp. 3.361 n. ${ }^{\circ} 5$.

15

1257, febrero', 2. Tortosa

Jaime I confirma a Umbertino de Lavolta la donación de las alquerias de Alboraya y Almácera y las casas de realengo de Valencia que hizo a su abuelo Sir Umberto de Lavolta. Pere Pau, notario, es testigo.

A.H.N. Clero. Valldigna. Bernardos. Carp. 3.361 n. ${ }^{\circ} 8$

REG. BARRAGAN GOMEZ, M. doc. 17

16

1257, julio, 6. Valencia

Guillermo Zamora vende unas casas que posee a Pedro Marbres. Guillem de Jaca, notario.

A.R.V. Caja 3, perg. 15.

17

1258, junio, 28. Valencia

Guillermo Salelles concede licencia para que su vecino, Pedro Coramis, pueda utilizar para lo que quiera el tránsito existente entre sus casas. Pere Pau notario.

A.R.V. Caja 3, perg. 16.

18

1259, enero, 10. Valencia

Arnau de Romaní establece en sus tierras de Ollería (Valencia) a Pere Ballester. Arnau de Llusâ, notario.

A.H.N. Clero. Valencia. Bernardos. Carp. 3.361 n. ${ }^{\circ} 13$. 
19

1259, marzo, 7. Valencia

Pedro Coromines y su mujer, Buenaventura, venden a Mateo de Carrera unas casas con sus huertos situadas en la Boatella junto al convento de la Merced de Valencia. Arnau Astruc, notario.

A.R.V. Caja 3, perg. 18.

20

1259, mayo, 11

Testamento de Lázaro Vilella, ciudadano de Valencia. Notario Guillermo de Jaca.

A.C.V. Perg. 1.330 .

REG. OLMOS CANALDA n. ${ }^{\circ}$

21

1260, abril, 5. Valencia.

Arnau de Romaní establece a Miquel Bategat en una tierra suya de Ollería (Valencia). Arnau de Llusâ, notario.

A.H.N. Clero. Valldigna. Bernardos. Carp. 3.361 n. ${ }^{\circ} 14$.

22

1260, agosto, 12. Valencia

Guillem de Villalonga y su mujer, Saurina, venden a Mateo de Cervera unas casas situadas en San Juan de la Boatella en la ciudad de Valencia. Arnau Astruc, notario.

A.R.V. Caja 3 n. 22.

23

1261, marzo, 19. Valencia.

Ramón de Roda vende un obrador a Jaime Gartellador. Arnau de Jaca, notario.

A.R.V. Caja 3 n. 26.

24

1261, abril, 5. Valencia.

Inés y Pedro Colent, y la mujer de este, Marsilla, venden a Jaime I unas casas y su columbario que están en Valencia, situadas junto al convento de Santa Magdalena. Arnau Astruc, notario.

A.R.V. Caja 3 n. $^{\circ} 25$.

\section{5}

1261, abril, 9. Valencia.

Trașlado del documento de venta que el 27 de agosto de 1245 realizó Abril de Fraga y su mujer a favor de Juan Colent y su mujer de unas casas y huertos situados en San Juan de la Boatella (Valencia). Arnau Astruc, notario.

A.R.V. Caja 3 n. $^{\circ} 24$. 
26

1262, marzo, 12. Valencia

Traslado del documento de confirmación de donaciones que el 2 de febrero de 1257 hizo en Tortosa el rey Jaime I a favor de Umbertino de Lavolta de las donaciones hechas a su abuelo, Sir Umberto de Lavolta. Arnau de Llusâ, notario.

A.H.N. Clero. Valldigna. Bernardos. Carp. 3.361 n. ${ }^{\circ} 8$.

REG. BARRAGAN GOMEZ, M. doc. 17.

27

1262, mayo, 3. Alzira

Pedro Lopez de Paracels vende unas tierras que posee en Carcasneu (Alzira) a Guillém de Castelló. Pere de Santa Creu, notario.

A.H.N. Clero. Valldigna. Bernardos Carp. 3.361 n. 18.

28

1263, mayo, 2. Valencia

Documento de confirmación de la venta de un censo entre Bernardo Meneri y Pedro de Guardia, vecino de Valencia. Arnau Astruc, notario. A.H.N. Clero. Merced. Puig. Carp. 3.362 n. ${ }^{\circ} 2$.

REG. VEDREÑO ALBA, M. ${ }^{a}$ C. $n .^{\circ} 48$.

29

1263, agosto, 10. Valencia.

Pedro Ballester y su mujer, Alicsenda, venden un huerto que tienen en Ollería (Valencia) en enfiteusis bajo Arnau de Romani a Jimeno Bateteri de Alcotano y su mujer. Arnau de Jaca, notario.

A.H.N. Clero. Valldigna. Bernardos. Carp. 3.362 n. ${ }^{\circ} 2$.

30

1264, febrero, 1. Alzira

Marco Galindo y Pedro Sanchez de Eslava, albaceas de Laura, mujer de Domenec de Cavall, venden unas casas situadas en Alzira a Simón Pedro de Arnedo y a su mujer.

A.H.N. Clero. Valldigna. Bernardos. Carp. 3.363 n. ${ }^{\circ} 5$.

31

1268, agosto, 25. Cervera.

Jaime I otorga potestad a Guillem de Rocafull para que pueda poblar veinte casas de sarracenos en Fortaleny. Pere Pau, notario, es testigo. Traslado de documento realizado por el notario Bernat Garcerà el 6 de marzo de 1270 .

A.H.N. Clero. Valldigna. Bernardos. Carp. 3.362 n. 18.

REG. BARRAGAN GOMEZ. M. Doc. 22. 


\section{2}

1272, octubre, 16. Valencia

Guillermina, mujer de Pedro Gilabert hace testamento ante el notario de Valencia Janer Rabaça.

A.C.V. Perg. 3.507.

REG. OLMOS CANALDA n.

33

1273, febrero, 26. Valencia

Jaime I otorga dominio por igual parte al obispo y cabildo sobre los lugares de Chulilla y Garig, zanjando así la querella entablado por el rey por razón de diezmos. Arnau Astruc, notario.

A.C.V. Leg. 12: 20.

REG. OLMOS CANALDA n. ${ }^{\circ} 472 a$.

34

1275, junio, 4. Valencia

Traslado del testamento de Pedro Marques. Pere Pau, notario. A.C.V. Perg. 2.917.

REG. OLMOS CANALDA n. 502.

35

1274, junio, 24. Valencia

Traslado del testamento de Pedro Abrafim realizado por el notario de Valencia Janer Rabaça del testamento original realizado por el notario Bertrán Galcerán el mismo día 24 de junio de 1274.

A.C.V. Perg. 1.350.

REG. OLMOS CANALDA n. ${ }^{\circ}$

36

1276, octubre, 21. Valencia

El rey Pedro III ordena a Guillém Colom, alcaide del castillo de Guadalest en nombre de Arnaldo de Mataró de que entregue aquel a Sancho Ortiz de Ruffets o a su escudero.

A.C.A. R. 38 f. 64.

REG. MARTINEZ FERRANDO, J. E. (I), n. ${ }^{\circ} 106$.

37

1279-80, enero, 7

Pedro III comanda la escribania de la ciudad de Valencia a Arnau Astruc durante la inhibición de los escribanos de dicha ciudad. A.C.A. R. 42 f. 200 v.

REG. MARTINEZ FERRANDO, J. E. (I) n. 799.

38

1279, noviembre, 21. Valencia. 
Testamento de Pedro Pérez, canónigo de la catedral de Valencia, por el que intituye un beneficio y un aniversario. Janer Rabaça, notario.

A.C.V. Perg. 1.362.

REG. OLMOS CANALDA $n .^{\circ}$

39

1281, enero, 16. Valencia

Arnau de Jaca, notario de Valencia, compra unas casas de dicha ciudad situadas en barrio de Xerea a Domingo Roselló y a Marta, su mujer. Guillermo de Agramunt, notario.

A.R.V. Caja 3 n. ${ }^{\circ} 31$.

40

1281, junio, 15

Mandato al justicia y baile de Valencia para que obliguen a Janer Rabaça y a Guiltermo Vernet a qe entreguen a Vidal y David Astruc las escrituras de los notarios de Valencia.

A.C.A. R. 50 f. 121 v.

REG. MARTINEZ FERRANDO, J. E. (l) n. ${ }^{\circ} 1.335$.

41

1281, diciembre, 5. Valencia

Arnau de Jaca, notario de Valencia, compra a Raimundo de Cervera y a su mujer unas casas situadas en la parroquia de San Esteban de Valencia. Janer Rabaça, notario.

A.R.V. Caja 3 n. 33.

42

1281, diciembre, 14. Valencia

Jaime de Monzon y su mujer venden a Juan de Monzon unas tierras que poseen en Olleria (Valencia) y que poseen en enfiteusis de Jaime de Romaní. Janer Rabaça, notario.

A.H.N. Clero. Valldigna. Bernardos Carp. 3.365 n. 3.

43

1282, febrero, 21. Valencia

Domenec de San Marti y su mujer venden a Juan de Monzon unas tierras que poseen en Olleria (Valencia) y que tienen en enfiteusis de Jaime de Romaní. Janer Rabaça, notario.

A.H.N. Clero. Valldigna. Bernardos. Carp. 3.365 n. ${ }^{\circ} 6$.

44

1286, junio, 7. Valencia

Jaime de Romaní reconoce en enfiteusis a Guillermo Català. Janer Rabaça, notario.

A.H.N. Clero. Valldigna. Bernardos. Carp. 3.365 n. ${ }^{\circ} 14$. 
1286, junio, 8. Valencia

Jaime de Romani reconoce la enfeteusis que tiene Domenèc Català en sus tierras de Olleria (Valencia). Janer Rabaça, notario.

A.H.N. Clero. Valldigna. Bernardos Carp. 3.365 n. ${ }^{\circ} 15$.

46

1286, junio, 9. Valencia

Jaime de Romani reconoce y renueva la enfiteusis que tiene Borras Steva en sus tierras de Ollería (Valencia). Janer Rabaça, notario. A.H.N. Clero. Valldigna. Bernardos. Carp. 3.365 n. ${ }^{\circ} 17$.

47

1287, marzo, 22. Valencia.

Testamento del obispo de Valencia Jazperto de Botonach ante el notario Janer Rabaça.

A.C.V. Perg. 5.565.

REG. OLMOS CANALDA $n .^{\circ}$

48

1288, agosto, 331. Alzira

Traslado del documento por el que Jaime I hace donación a Berenguer de Tamrit de unas casas en Alzira y otros lugares. Guillém Colom es testigo. Primer notario Pere Andreu. Notario del traslado Pere de Martorell, notario de Alzira.

A.H.N. Clero. Valldigna. Bernardos. Carp. 3.360 n. ${ }^{\circ} 1$.

REG. BARRAGAN GOMEZ, M. Doc. 10.

49

1289, enero, 27. Valencia.

El rey Alfonso III concede a Janer Rabaça, notario de Valencia, 150 sueldos anuales que recibirá sobre los reditos de la bailia de dicha ciudad.

A.C.A. R. 78 f. 37 .

REG. GALLOFRE GUINOVART, R. n. ${ }^{\circ} 1.244$.

50

1290, abril, 17. Valencia

Apoca que Pedro de Barberá firma a favor de Janer Rabaça, notario, por la compra de unos censos.

A.C.V. Perg. 4.692.

REG. OLMOS CANALDA $n$. $^{\circ}$

51

1290, abril, 18. Valencia

Documento por el que Pedro de Barberá reconoce a Janer Ra- 
baça, notario, las rentas de unos censos.

A.C.V. Perg. 1.379.

REG. OLMOS CANALDA $n .^{\circ}$

52

1290, agosto, 8. Valencia.

Alfonso III comunica al Justicia y a los Jurados de la ciudad de Valencia que no manden nada a Janer Rabaça, notario, ya que el le necesita.

A.C.A. R. 81 f. 147 v.

REG. GALLOFRE GUINOVART, R. n. ${ }^{\circ} 1.649$.

53

1291, diciembre, 10. Valencia

Ramón Falcó y su mujer, Maria, venden a Esteve Planes un huerto que poseen en Museros. Janer Rabaça, notario.

FONDO CRESPI DE VALLDAURA, n. ${ }^{\circ} 4$.

54

1291, diciembre, 12

Documento por el que Jaime II nombra notario de Requena a Pere Pau.

A.C.A. R. 195 f. 106.

55

1292, octubre, 23. Valencia

Pedro Ribalta y su mujer venden a Pedro Becep unas casas situadas en la parroquia de San Andrés de Valencia. Janer Rabaça, notario.

A.R.V. Caja 3 n. 38.

56

1295, enero, 17. Valencia.

Documento de posesión de bienes realizado por Guillém de Jaca, notario.

A.P.V. Protocolo de Pedro Montull, año 1430 n. ${ }^{\circ} 1.595$.

57

Raimunda Aulas, hija de Bernardo Aulas, vende unas casas situadas en San Juan de la Boatella (Valencia) a Bernardo Clavell. Están sujetas a censo de Arnaldo de Monroig. Janer Rabaçz, notario.

A.R.V. Caja 3 n. 42. 